\title{
CONSECUENCIAS DEL FIN DE LA HEGEMONÍA ESTADOUNIDENSE EN LA GERENCIA LATINOAMERICANA
}

\author{
Sealtiel Álvarez Sánchez \\ International Business Machines (IBM), Costa Rica \\ salvarez@racsa.co.cr
}

\section{RESUMEN}

Después del final de la Segunda Guerra Mundial, Estados Unidos emergió como una potencia mundial. Aunque esta nación sigue siendo protagonista de la economía mundial, los recientes acontecimientos mundiales parecen haber debilitado su posición hegemónica. El dólar pierde terreno en relación con el euro, en parte como resultado de la recesión económica mundial. Mientras tanto, algunas monedas asiáticas se fortalecen. India y China están emergiendo como nuevas potencias económicas mundiales. El mundo está cambiando hacia una situación multipolar sin multilateralismo. Estos cambios estructurales tienen importantes implicaciones en el trabajo de los gerentes latinoamericanos al menos en las siguientes áreas: reinserción de las economías locales en la economía internacional, reformulación de las relaciones económicas con Estados Unidos y los grandes consorcios internacionales, asimilación de la tecnología moderna y formulación de políticas para la gestión del talento humano.

PALABRAS CLAVE: HEGEMONÍA ESTADOUNIDENSE, GERENCIA LATINOAMERICANA, ECONOMÍA GLOBALIZADA

\section{ABSTRACT}

After the end of the Second World War, the United States emerged as a world power. Although this nation is still a protagonist in the world economy, recent worldwide events seem to have weakened its hegemonic position. The dollar is losing ground against the euro, partly as result of the world economic recession. Meanwhile, other Asian currencies are gaining strength. In these conditions India and China are emerging as new economic world powers. The world is moving towards a multi-polar situation without multilateralism. These structural changes have important implications in the work of the LatinAmerican managers in at least the following areas: reintegration of local economies in the international economy, reformulation of economic relations with the United States and large international enterprises, integration of modern technology and formulation of human resource management policies.

KEYWORDS: AMERICAN HEGEMONY, LATIN-AMERICAN MANAGEMENT, GLOBALIZED ECONOMY.

\section{Introducción}

El denominado proceso de globalización de la economía ha sido históricamente liderado por Estados Unidos. Desde el final de la Segunda Guerra Mundial esta nación ha ejercido gran influencia mundial en todos los ámbitos: comercial, económico, político y militar. Su influencia se ha extendido hasta aspectos culturales y ha influenciado en la forma en que se visten y alimentan los ciudadanos de diversas naciones. Algunos autores han llamado a esta influencia un "nuevo orden internacional". En 1992 Flores (1992) trató el tema desde la perspectiva de los derechos humanos, el desarrollo de la tecnología, la carrera armamentista y la influencia ideológica de Estados Unidos en el mundo.

Sin embargo, algunos acontecimientos mundiales parecen estar cambiando el panorama: la reciente crisis económica ocasionada principalmente por los problemas hipotecarios en Estados Unidos; el surgimiento de nuevas potencias económicas, especial- 
mente India y China en Asia y Brasil en América y el debilitamiento del dólar en relación con el euro parecen tener una importante influencia en la posición hegemónica de Estados Unidos a nivel mundial.

Dado que Estados Unidos es el principal socio comercial de la mayoría de los países latinoamericanos un cambio en su posición hegemónica influye en las estrategias país de las demás naciones. Entonces, resulta primordial que la dirección estratégica de las empresas se adapte a estas novedades estructurales.

A través de este ensayo se pretende que el lector adquiera un entendimiento más amplio de los factores que influyen en este cambio mundial así como de las implicaciones que éste tiene para el gerente latinoamericano, en especial en lo relacionado con la formulación de la estrategia comercial.

\section{Desde Occidente hacia Oriente}

A esta generación le ha tocado presenciar cambios transcendentales en la economía mundial. A partir, de la década del ochenta con los gobiernos estadounidenses de Reagan, Bush padre, Clinton y Bush hijo, la nación del norte se acostumbró a dar cátedra al resto del mundo sobre cómo mantener en orden las finanzas públicas.

Sin embargo, resultó paradójico que mientras Estados Unidos explicaba al resto de las naciones como mantener sus finanzas saludables, a lo interno descuidaron su economía permitiendo que sugiera una crisis como hace décadas no experimentaba esa nación. Esta condición les restó autoridad moral para aconsejar otros países sobre la manera más apropiada de administrar sus finanzas. También perdieron credibilidad internacional cuando durante la Guerra en Irak fueron en busca de armas nucleares y químicas, sin nunca encontrarlas en forma fehaciente.

En las actuales condiciones económicas, resulta de gran interés entender los cambios económicos mundiales y el surgimiento de nuevas economías.
En términos del crecimiento real del Producto Interno Bruto (PIB), ¿cuál fue la situación durante 2009 y qué expectativas hay para el 2010? Estudios del Fondo Monetario Internacional (FMI) sugieren que nuevas potencias económicas están surgiendo en el contexto internacional. Por ejemplo desde 2008 la República Popular China es la segunda potencia económica mundial de acuerdo con el valor nominal de su PIB. A pesar de la recesión económica mundial el FMI proyecta un crecimiento real del PIB de China del 8,5\% en 2009 y del 9\% en 2010. El mismo informe proyecta un crecimiento para India de 5,4\% y 6,4\% respectivamente. Se pronostica un crecimiento del 3,5\% en el PIB brasileño para el 2009, mientras que el PIB del 2010 decrecerá en un 0,7\%. En México el indicador decrecerá un 7,3\% en el 2009 y se espera que crezca a un ritmo del 3,3\% en el 2010. ¿Cuál es la situación en Estados Unidos? Se espera que el PIB de Estados Unidos decrezca un 2,7\% en el 2009 mientras en el 2010 se espera que crezca solamente un 1,3\%. (FMI, 2009).

Las cifras anteriores parecen confirmar la opinión de Nebot et al. (2006) de que la riqueza se está desplazando desde occidente hacia oriente. Se espera que el continente asiático emerja con fuerza en las próximas décadas no solo en términos económicos, sino incluso tecnológica y culturalmente. Sin embargo, este desarrollo económico no ocurre sin implicaciones ambientales. Por ejemplo, China experimenta altos niveles de contaminación en sus principales ciudades en tanto Estados Unidos con solo el 4\% de la población mundial es uno de los principales emisores de gases contaminantes del mundo. Esta condición motivó que Estados Unidos se retirara del Protocolo de Kioto en el año 2001, argumentando que su aplicación es injusta e ineficiente.

¿Cuáles son las expectativas para Latinoamérica? Estudios del FMI predicen que Brasil ganará terreno e influencia mundial, probablemente por encima de México. Esto ocurrirá en parte debido al gran tamaño de la economía brasileña y la diversificación de sus productos y mercados, especialmente 
relacionada con el aumento de las relaciones con Asia. Es de esperar que exista en la región latinoamericana un costo ambiental asociado con el crecimiento económico, probablemente coherente con la escasez de agua potable y reducciones en la productividad de las cosechas (FMl, 2009).

\section{Multipolaridad sin multilateralismo}

En las actuales condiciones mundiales es poco probable que durante las siguientes décadas una sola nación tenga amplia influencia a nivel mundial. La Dirección Nacional de Inteligencia del Gobierno de los Estados Unidos (2008) prevé que las coaliciones de naciones tomen gran importancia. No es necesario esperar años para ver esta situación. Desde el punto de vista comercial cada vez es más común que las naciones conformen grupos económicos para negociar tratados comerciales. El Tratado de Libre Comercio entre República Dominicana - Centroamérica - Estados unidos, más conocido como CAFTA-DR por sus siglas en inglés, es un ejemplo de esta tendencia. La misma situación se percibe en las negociaciones que realiza Centro América con la Unión Europea. Estas condiciones plantean un mundo multipolar. La generación actual está viviendo un histórico período de cambio.

Las organizaciones internacionales incluyendo las ONGs, movimientos sociales, organizaciones filantrópicas, organismos multilaterales y grandes compañías multinacionales han tomado relevancia mundial. Se prevé que esta fragmentación de intereses y actores dificulte la posibilidad de llegar a acuerdos multilaterales en una forma sencilla. Los líderes mundiales buscarán alternativas para resolver los grandes problemas transnacionales, incluido el cambio climático, regulación de los mercados financieros globales, migración y redes criminales internacionales (Dirección Nacional de Inteligencia del Gobierno de los Estados Unidos, 2008).

McGarvie (2009) menciona algunos factores clave que mueven el mundo multipolar en el cual vivimos:
- Nuevos mercados de consumidores. Se espera que los consumidores de mercados como China, India y México representen el 50\% del consumo mundial para 2025. Entonces, es necesario desarrollar productos y servicios innovadores para estos nuevos mercados mundiales.

- Atracción de capitales multidireccionales. En la nueva economía mundial las inversiones de las grandes compañías multinacionales representarán oportunidades de crecimiento para los países latinoamericanos. Sin embargo, estas inversiones no provendrán exclusivamente de compañías estadounidenses, sino incluirá a consorcios de las nuevas potencias económicas.

- Nuevo mapa de innovación tecnológica. Clústeres de innovación tecnológica están surgiendo en nuevas economías, tales como Beijing, Bengalooru y Krakow. Por ejemplo, en China se gestan el $12 \%$ de las patentes mundiales de nanotecnología. Las nuevas potencias económicas están evolucionando de "imitadores tecnológicos" a gestores en investigación y desarrollo (I+D).

- $\quad$ Desarrollo del talento. Para el año 2050 el 97\% de la fuerza laboral mundial vendrá de economías en desarrollo, incrementando en forma significativa la competencia por el talento. Esto representa un reto para los procesos de selección y reclutamiento de las empresas, así como para sus programas de capacitación.

Estas condiciones incrementan el riesgo de hacer negocios internacionales. Los factores de riesgo e incertidumbre se han incrementado con el tiempo principalmente debido a las diferencias culturales y económicas que Estados Unidos ha liderado en el contexto internacional. Sin embargo, en el mundo multipolar, donde existen dificultades para tomar acuerdos multilaterales, es posible que la influencia de esta nación sea menor. El desarrollo de las economías emergentes, como China, India, Brasil y Rusia determinará hasta qué grado Estados Unidos continuará imponiendo su política. 
El gerente latinoamericano realiza sus actividades mercantiles en medio de este deleznable ambiente. No obstante, al mismo tiempo que las actuales condiciones mundiales representan un reto, son también una oportunidad para los emprendedores. ¿Cómo puede el gerente latinoamericano obtener beneficio de estas nuevas condiciones mundiales? Recientemente varios autores han ofrecido ideas interesantes sobre estrategias que pueden ser de utilidad para incorporar la empresa en forma exitosa en los nuevos mercados.

\section{Gerencia Latinoamericana en un mundo multipolar}

Debemos considerar un mundo donde India está creciendo rápidamente y China es la segunda potencia económica mundial con Estados Unidos aportando solo un 2\% del PIB mundial. (McGarvie, 2009). Durante las pasadas décadas el poder se concentró principalmente en manos estadounidenses; sin embargo, estamos entrando en un período de crecimiento económico dominado por economías no-estadounidenses.

Existen una serie de acciones que deben considerarse para adaptar la organización a los cambios estructurales que presenciamos. A continuación, se presentan algunas estrategias que el gerente latinoamericano debe analizar concienzudamente si desea que su empresa sobreviva en el nuevo orden económico mundial.

\section{Enfocado, pero sin un negocio único}

Gary Hamel y Breen Bill (2007) presentaron una idea revolucionaria en el afamado editorial Harvard Business Press. Según ellos una compañía debe ofrecer cierto grado de libertad con el propósito de que sus colaboradores busquen intereses propios y potencien sus habilidades de forma tal que se beneficie toda la compañía. En la práctica esto significa estar abiertos a nuevos mercados y nuevos productos.
Esta apertura a nuevos productos y mercados es fundamental. Por ejemplo, cuando IBM perdió una parte importante de su liderazgo en el mercado de hardware se vio ante la necesidad de reinventarse a sí misma. Louis Gerstner llegó a la compañía cuando se consideraba que el core business de IBM era obsoleto. Entonces, se presentó una idea innovadora: competir en el mercado de los servicios tecnológicos. Ese fue uno de los grandes aciertos de Gerstner. Actualmente esa actividad representa aproximadamente el 50\% de los ingresos de esa compañía. De forma similar, algunas empresas costarricenses han gestionado procesos novedosos. Por ejemplo Café Britt implementó programas de "innovación irreverente" que modificaron su negocio tradicional, haciéndolo más rentable y competitivo en las actuales condiciones económicas mundiales.

Históricamente los países latinoamericanos han basado su economía en ciertos productos y servicios tradicionales, como el café y banano en el caso de Costa Rica. Si bien es cierto que esta situación está cambiando paulatinamente es necesario que el gerente latinoamericano asegure la sostenibilidad de la demanda y los precios en el largo plazo.

La economía mundial se está moviendo hacia la producción de bienes y servicios con gran valor agregado, algunos de corte tecnológico. Para incorporarse en la actual economía internacional es necesario que las organizaciones revalúen los bienes y servicios que ofertan en el mercado.

La demanda de servicios a nivel mundial representa una interesante oportunidad para las empresas latinoamericanas. El crecimiento de los servicios financieros, de salud, recreación y estética son ejemplos de algunas posibilidades innovadoras para Latinoamérica. Sin embargo, no es sencillo cambiar los procesos actuales para aprovechar estas oportunidades ya que en general las empresas de esta región enfrentan limitaciones financieras. Por esta razón el gerente latinoamericano debe enfocarse en la comercialización de opciones con alto valor agregado. 
En un mundo multipolar, donde cada vez el poder del cliente es mayor, resulta fundamental que las empresas latinoamericanas sean capaces de innovar en productos, servicios y procesos para generar nuevas oportunidades de negocios, diferentes de las tradicionales. Eso le facilitará a la empresa reinsertarse en la nueva economía mundial.

\section{Reformulación de las relaciones económicas con Estados Unidos y los grandes consorcios internacionales}

En los últimos años las exportaciones latinoamericanas hacia los países asiáticos han aumentado. Según informe de la Comisión Económica para América Latina y el Caribe (CEPAL, 2005) los mercados asiáticos se convirtieron en otro destino para las exportaciones latinoamericanas. Principalmente se exportan productos agrícolas, energéticos y químicos. Con esto se ha logrado compensar la baja en los envíos a Estados Unidos y Europa.

El gerente latinoamericano debe analizar las posibilidades de establecer relaciones comerciales con las nuevas potencias económicas mundiales. A nivel de pequeñas y medianas empresas se realizan muy pocas exportaciones, en la mayoría de los casos es menos de un 10\% las PYMES que realizan exportaciones. Es un desafío para las empresas en Latinoamérica expandir sus mercados hacia otras regiones diferentes de Estados Unidos. Los gobiernos de los países tienen instituciones que ofrecen asesoría para iniciar o reformular la estrategia exportadora.

Las empresas latinoamericanas pueden sacar beneficio de la fragmentación de intereses, característica del mundo multipolar. Especialmente para las pequeñas y medianas empresas latinoamericanas existe una importante oportunidad de negocios en medio de estas circunstancias. Muchas organizaciones pequeñas y medianas se han convertido en proveedores de bienes y servicios de las grandes multinacionales.

Jansson (2007) propone un enfoque de redes para enfrentar estas condiciones. El nuevo ambiente mundial se caracteriza por la fragmentación de intereses y la necesidad de operar en forma simultánea en varias regiones y sectores económicos. En este ambiente institucionalizado el autor propone establecer relaciones cooperativas a largo plazo con las principales firmas y sectores económicos, en especial con aquellas que pertenecen a las naciones emergentes. Estas redes de intereses comunes son una estrategia comercial que se adapta a las características del mundo multipolar.

Es posible implementar esta estrategia mediante el establecimiento de relaciones de partnership con estas grandes corporaciones. El partnership es un acuerdo comercial mediante el cual dos organizaciones comparten las ganancias o pérdidas de un determinado negocio. Muchas grandes corporaciones favorecen este tipo de acuerdos con propósitos fiscales.

El gerente latinoamericano tiene el desafío de buscar nuevas alianzas comerciales con las nuevas potencias económicas y los grandes consorcios internacionales. Bajo ciertas legislaciones el partnership es un excelente método para incursionar en nuevos mercados. Tal es el caso de China y de aquellos países donde aplican la ley islámica y donde la figura de las empresas constituidas completamente por capital extranjero está limitada o prohibida por la cultura o la ley vigente.

\section{Incorporación de tecnología en la estrategia comercial}

El avance tecnológico es una característica propia de la época moderna. Los gerentes latinoamericanos tienen dos posibilidades para incorporar nueva tecnología a sus procesos productivos: a) desarrollar tecnología propia o b) adquirida. La segunda opción se adapta mejor a las pequeñas y medianas empresas que tienen limitaciones financieras. Las grandes corporaciones latinoamericanas probablemente estén en capacidad de realizar mayores inversiones en I+D. 
La incorporación de tecnología en las actividades empresariales es fundamental para aumentar la competitividad en las organizaciones. En la mayoría de los países latinoamericanos existen redes tecnológicas de apoyo. En algunos casos los gobiernos ofrecen asesoría al respecto.

Un aspecto interesante de esta tendencia es el uso del software libre en las organizaciones públicas y privadas. El gerente latinoamericano puede encontrar en el uso de software libre una excelente oportunidad para tener acceso a tecnología de calidad a precios muy reducidos. Existen en el mercado muchas posibilidades para implementar sistemas ERP (Enterprise Resourse Planning) mediante software libre. Tal es el caso del sistema openXpertya, que es un proyecto financiado por el gobierno español.

Una tendencia novedosa de los mercados es el uso del E-commerce. Algunos modelos comunes son el B2B, B2C y C2C. Se estima que las ventas hechas mediante el comercio electrónico B2C crecerán a una tasa del 23.3\% alcanzando 168 billones de dólares en el 2011, solo en la región de Asia Pacífico. Para este año se estima que China e India serán los mercados de e-commerce más grandes del mundo (Trends in the Global E-Commerce Market, 2008).

El uso de la tecnología en los procesos productivos se estima de gran importancia ya que los mercados emergentes como China, India y algunos otros pocos países asiáticos han sido tradicionalmente sensibles a los precios. La tecnología representa una excelente oportunidad para probar la habilidad de las empresas latinoamericanas para proveer productos y servicios a estos mercados a precios competitivos.

\section{Formulación de políticas innovadoras para gestionar el talento humano}

En las actuales condiciones económicas no es extraño que el gerente latinoamericano afronte dos posibles escenarios. Primero, es posible que enfrente situaciones dicotómicas y se vea ante la disyuntiva de elegir entre el uso de tecnologías y procesos que ahorran mano de obra. Segundo, es muy probable que se vea ante la necesidad de utilizar talento humano con diferentes orígenes culturales y étnicos.

Estudios realizados por la Comunidad Europa han demostrado que uno de los problemas que enfrentan las pequeñas y medianas empresas está relacionado con el acceso a recurso humano capacitado, principalmente debido al elevado costo de los mismos. El Observatorio de PYMES Europeas confirmó en el 2007 que el 5\% de las vacantes en las PYMES europeas permanecía sin Ilenar principalmente por la escasez de mano de obra calificada (28\%) y el elevado costo de los recursos disponibles (33\%) en el mercado (Observatorio de PYMES Europeas, 2007). En América Latina existe una situación similar. Por esta razón es necesario que el gerente de esta parte del mundo genere estrategias que permitan desarrollar las habilidades requeridas al menor costo posible. Entonces se hace imprescindible la incorporación de programas de capacitación para desarrollar los recursos propios con el propósito de que estos ocupen posiciones de responsabilidad dentro de la organización.

En la mayoría de los países de Latinoamérica existen opciones de capacitación de bajo costo o gratuitas que pueden usarse como parte del programa de gestión del talento humano. Internet ofrece abundante material para desarrollar procesos de capacitación. El e-learning avanza en forma vertiginosa. Stephen Downer es un renombrado experto en esta opción educativa. El dice que"resurgirán las comunidades en línea y aparecerán los recursos de aprendizaje, libres y gratuitos, convirtiendo a la Red de redes en una alternativa viable de la educación tradicional" (E-Learning América Latina, 2004, p. 1). Un análisis exhaustivo de estas opciones le permitirá a la empresa latinoamericana con limitados recursos financieros obtener capacitación de calidad y desarrollar el personal para enfrentar los retos del actual ambiente económico mundial. 
En relación con la contratación de recursos internacionales con diversos antecedentes culturales, Lionel Laroche y Don Rutherford (2007) reconocen es un reto y una oportunidad. Es necesario que las empresas adapten sus sistemas de reclutamiento y selección para hacer uso de las recientes tecnologías disponibles, en particular Internet. El gerente latinoamericano debe familiarizarse con formas de comunicación verbal y no verbal que podrían resultar desconocidas anteriormente y que son propias de cada cultura. Lograr esa"conexión cultural"es fundamental para obtener el éxito. Es necesario revisar también las políticas de compensación y el paquete de beneficios con el propósito de retener a aquellos empleados más calificados que realizan aportes significativos al negocio. Aunque no es fácil lograr lo anterior, el gerente debe reconocer que usualmente los beneficios vienen después de los retos.

\section{CONCLUSIONES}

La posición hegemónica de los Estados Unidos se ha visto debilitada por el surgimiento de nuevas potencias económicas en Asia y por el efecto de la recesión económica mundial. La principal consecuencia de este debilitamiento es el establecimiento de un mundo multipolar, donde el establecimiento de acuerdos multilaterales es cada vez más difícil. Esta fragmentación de intereses ha tenido como resultado un mayor protagonismo de las organizaciones internacionales como las ONGs y las grandes compañías multinacionales.

Estas condiciones representan un reto para el gerente latinoamericano, pero al mismo tiempo constituyen también una oportunidad de negocios. Es necesario que se reformulen las relaciones económicas con los Estados Unidos y las grandes corporaciones al mismo tiempo que se realizan esfuerzos concretos para reinsertar las empresas en la economía mundial mediante el uso de la tecnología y eficientes políticas para la gestión del talento humano pluricultural.
Mediante estas acciones las empresas latinoamericanas podrán sacar beneficio del cambio en las condiciones mundiales. Como siempre, los responsables de las empresas son quienes al final fijan el rumbo de las mismas e influyen en su éxito o fracaso. La decisión es suya.

\section{REFERENCIAS}

Comisión Económica para América Latina y el Caribe. (2005). Economic indicators of Latin America and the Caribbean. Santiago, Chile: Division of International Trade and Integration:

Dirección Nacional de Inteligencia del Gobierno de los Estados Unidos. (2008). Global Trends 2025: A Transformed World. Noviembre 2008. Washington, DC : Superintendent of Documents, US Government Printing Office.

E-Learning América Latina. (2004) ¿Hacia dónde va el e-learning? Revista Digital de E-Learning América Latina. 1 de febrero de 2004. Recuperado el 09-10-2009 de http://www.elearningamericalatina.com/edicion/febrero1_2004/na_1.php

Flores, C. T. (1992). ¿Un nuevo orden internacional? Una introducción a los problemas internacionales en el final del siglo. Madrid, España : Ediciones La Torre.

Fondo Monetario Internacional (octubre, 2009). World Economic Outlook: a survey by the staff of the International Monetary Fund. Washington, DC: International Monetary Fund.

Hamel, Gary \& Bill Breen. (2007). The Future of Management. Boston, Massachusetts, Harvard Business Press Estados Unidos.

Jansson, H (2007). International Business Strategy in Emerging Country Markets: The Institutional Network Approach. Northampton, Massachusetts: Edward Elgar Publishing.

Laroche, Lionel y Don Rutherford. (2007). Recruiting, Retaining, and Promoting Culturally Different Employees. Butterworth-Heinermann: Massachusetts, Estados Unidos. 
Nebot, P.; Seager, P.; Carr, K. y Vila, J.M. (setiembre, 2006). La era de las nuevas potencias económicas. Revista de Antiguos Alumnos 37, 52-59. Universidad de Navarra : Navarra, España.

Observatorio de PYMES Europeas (2007). Reporte Analítico 2007. Flash Eurobarometer. Comisión Europea.

McGarvie, B. J. (2009). Shaking the Globe: Courageous Decision-Making in a Changing World. Hoboken, New Jersey, Estados Unidos: John Wiley \& Sons.

Trends in the Global E-Commerce Market. (2008). Consensus Report. Analyst Perspectives. Recuperado el 10-10-2009 de: http://common.books24x7.com/book/id_28617/book.asp (10 de octubre de 2009)

RECIBIDO: 22-10-2009

ACEPTADO: 04-12-2009 